Abstracts: Session II

prometaphase chromosomes. Linkage to existing physical maps is provided by the requisite presence of a mapped sequence tagged site in all selected BACs and in many cases by the full or end sequencing of the mapped clone itself. Clones in the repository should allow all chromosomal breakpoints to be localized to within 2 $\mathrm{Mb}$ and provide entry points to existing physical maps of the intervening distance. With the completion of a rough draft of the human genome, sequences between mapped breakpoints can be immediately queried for potential candidate genes. We will provide an update on the number of BAC clones and chromosomes currently mapped and available to the biomedical community. One critical issue is the integration of databases for the cytogenetic and physical maps. The National Cancer Institute and National Center for Biotechnology Information are working together to develop a meaningful integration that provides (1) a direct connection between catalogued and newly discovered chromosomal breakpoints or regions of genomic imbalances and the BAC clone set; (2) direct display of recurring chromosomal breakpoints that coincide with BAC locations; (3) placement of mapped BACs on the human sequence; (4) tools to build contiguous clone sets; (5) integration of the BAC clone set with databases for comparative genomic hybridization and spectral karyotyping and (6) an interface for the identification of orthologous regions of chromosomal aberrations in human cancers and their respective mouse models.

Klein, Christoph [29]

\section{Transcriptome and genome analysis of single disseminated tumor cells: approach to study minimal residual cancer}

Christoph Klein ${ }^{1}$, Stefan Seidl ${ }^{1}$, Karina Petat-Dutter ${ }^{1}$, Oleg Schmidt-Kittler ${ }^{1}$, Sonja Offner ${ }^{2} \&$ Gert Riethmüller $^{1}$

${ }^{1}$ Institut für Immunologie, Ludwig-Maximilians-Universität, Munich, Germany ${ }^{2}$ Micromet GmbH, Martinsried, Germany

Despite increasing molecular-genetic understanding of the development of malignant epithelial neoplasias, the front-line therapy for patients with carcinomas is still surgery. Because systemic adjuvant treatments such as chemotherapy or immunotherapy have had limited success and because the characteristics of systemically disseminated tumor cells can be very different from those of the primary tumor or end-stage metastasis, we have studied the evolution and progression of systemic cancer directly. We have developed techniques to detect, isolate and study genomes and transcriptomes of single micrometastatic cells present in bone marrow of carcinoma patients. The first results of our genetic analysis demonstrate that disseminated tumor cells are clonally related, indicating their selection from heterogeneous cell populations of the primary tumor. Gene expression analysis of single disseminated tumor cells revealed their engagement in specific functional activities, such as tissue invasion, proliferation and DNA damage repair. Detailed analyses of single disseminated cells will help determine which genotypes and phenotypes are selected during dissemination, which cells survive in the new environment and what governs the establishment of a metastasis. This knowledge will be useful in selectively targeting the precursor cells with adjuvant therapies long before metastatic disease is clinically evident and incurable.
Krahe, Ralf

$[30]$

\section{Gene expression profiling reveals fundamental biological differences in AML with trisomy 8 and normal cytogenetics}

Kimmo I. Virtaneva ${ }^{1}$, Fred A. Wright ${ }^{1}$, Stephan M. Tanner ${ }^{1}$, Bo Yuan ${ }^{1}$, William J. Lemon ${ }^{1}$, Michael A. Caligiuri2,3, Clara D. Bloomfield ${ }^{2,3}$, Albert de la Chapelle ${ }^{1} \&$ Ralf Krahe $^{1}$

${ }^{1}$ Division of Human Cancer Genetics, Department of Molecular Virology, Immunology and Medical Genetics, Comprehensive Cancer Center, Ohio State University, Columbus, Ohio 43210, USA

${ }^{2}$ Division of Hematology/Oncology, Department of Internal Medicine, Comprehensive Cancer Center, Ohio State University, Columbus, Ohio 43210, USA ${ }^{3}$ Cancer and Leukemia Group B, Chicago, Illinois 60604, USA

Acute myeloid leukemia (AML) is a heterogeneous group of diseases. Patients exhibiting normal cytogenetics (AML-CN) constitute the single largest group, and trisomy $8(\mathrm{AML}+8)$ as the sole abnormality is the most frequent trisomy. How trisomy contributes to tumorigenesis is unknown. Because hematopoietic differentiation is predominantly regulated at the transcriptional level, we proposed that, whatever the underlying molecular leukemogenic event(s) associated with AML$\mathrm{CN}$ and $\mathrm{AML}+8$, the molecular changes at the DNA level should be reflected in specific changes at the RNA level. We used oligonucleotide-based DNA microarrays to study global gene expression in ten $\mathrm{AML}+8$ patients with +8 as the sole chromosomal abnormality and ten AML-CN patients, as well as seven CD34+ cell samples purified from normal bone marrow of healthy individuals as a representative heterogeneous population of stem and progenitor cells. For the 6,606 genes studied, expression patterns of AML patients were clearly distinct from those of CD34+ cells of normal individuals. AML +8 was associated with an overexpression of genes on chromosome 8 , suggesting a role for gene dosage effects in the etiology of AML +8 . Systematic analysis by cellular function indicated upregulation of genes involved in cell adhesion in both AML groups. We observed other highly significant results in the comparison of $\mathrm{AML}+8$ with AML-CN for genes involved in the regulation of apoptosis, suggesting a fundamental biological difference in programmed cell death. The observed differences in the dysregulation of specific functional subsets of genes between the two AML subclasses merit further functional studies of the individual components of the pathways.

Krupke, Debra M.

[31]

\section{Electronic access to data from mouse cancer models: The Mouse Tumor Biology database}

Debra M. Krupke, Carol J. Bult, Dieter Naf, John P. Sundberg \& Janan T. Eppig

Jackson Laboratory, 600 Main Street, Bar Harbor, Maine 04609, USA

The Mouse Tumor Biology database (MTB) has been developed to provide electronic access to mouse tumor biology data. The primary focus of MTB is spontaneous and induced tumors in genetically defined mice (inbreds, hybrids, mutants and genetically engineered strains). By making such data readily available online, MTB provides the scientific community with a much-needed, easily accessible central resource for rapidly finding and evaluating the expanding volume of mouse tumor data. MTB provides cancer researchers with access to data on mouse models for cancer and includes information such as tumor names and classifications, pathology reports, histopathological images, genetics of the strain, genomic changes in the tumor, strain names, tumor frequency and latency, references and 


\section{Abstracts: Session II}

links to other related online resources. The MTB database is designed to facilitate the selection of experimental models for cancer research, the evaluation of mouse genetic models of human disease, the review of patterns of mutations in specific cancers and the identification of genes that are commonly mutated across a spectrum of cancers. Recent enhancements to MTB include a redesigned Web interface for more powerful querying and improved views that simplify and better organize data presentations. A significantly expanded archive of histopathological images also is available. The MTB prototype database is accessible at http://tumor.informatics.jax.org. User support is available by e-mail to mgi-help@informatics.jax.org.

Kuzumaki, Noboru

\section{Epigenetic silencing of the tumor- suppressing gelsolin gene involves histone deacetylation of its promoter region in human urinary bladder cancer}

Kazunori Haga ${ }^{1,2}$, Hisakazu Fujita ${ }^{1}$, Ataru Sazawa ${ }^{1,2}$, Masato Takimotoㄹ, Nobuo Shinohara ${ }^{2}$, Tomohiko Koyanagi ${ }^{2}$ $\&$ Noboru Kuzumaki ${ }^{1}$

${ }^{1}$ Division of Cancer Gene Regulation, Institute for Genetic Medicine, Hokkaido University, Hokkaido, Japan

${ }^{2}$ Department of Urology, School of Medicine, Hokkaido University, Hokkaido, Japan

Alterations in histone acetylation seem to play a central role in the repression of several important genes, such as tumor suppressor genes. Previously we reported that gelsolin expression was reduced in human urinary bladder cancers and that ectopically expressed gelsolin acted as a tumor suppressor ${ }^{1}$. We have examined the effects of trichostatin A (TSA), a specific histone deacetylase inhibitor, to examine the histone acetylation status of the gelsolin promoter region in bladder cancers. We measured gelsolin protein expression by western blotting in bladder cancer cell lines treated with various doses of TSA. We assessed promoter activities of the gelsolin gene by luciferase assays in both nontreated and TSA-treated bladder cancer cell lines. The acetylation status of histones linked to the gelsolin promoter was checked using chromatin immunoprecipitation and a dot blot assay. Gelsolin protein production was much reduced in cancer cell lines. Both re-expression and the promoter activity of gelsolin induced by TSA were dose- and time-dependent. An antibody that reacts with acetylated histones $\mathrm{H} 3$ and $\mathrm{H} 4$ immunoprecipitated chromatin containing the gelsolin promoter in the TSA-treated bladder cancer cells, but not in nontreated cells. These results suggest that TSA activated the gelsolin gene promoter through histone acetylation. In bladder cancer, the repression of the gelsolin gene is related to the deacetylation of histones to its promoter region. Together with the reported epigenetic changes in histone acetylation in breast cancers, acetylation-mediated gene silencing could be a common mechanism of gelsolin downregulation in several cancers.

1. Haga, K. et al. Cancer Res. 55, 3228 (1995).
Lacher, Markus

[33]

\section{Wnt-Frizzled signaling in cell proliferation and survival}

\author{
Markus Lacher \& Robert Friis \\ Department of Clinical Research, University of Berne, Berne, Switzerland
}

We have used the differential display method as a coincidence analysis technique to isolate genes expressed in different rat tissues undergoing physiological apoptosis, such as involuting mammary gland or prostate after castration. One isolate, $D D C-4$, encodes a secreted Frizzled-Related Protein of $39.7 \mathrm{kD}$ in an open reading frame of 1,044 nucleotides. This protein family is thought to antagonize the signaling of Frizzled transmembrane receptors. Inappropriate expression of $D D C-4$ in lactating mammary glands of transgenic mice induces apoptosis during late pregnancy and early lactation. $D D C-4$ presumably acts by blocking a survival pathway. Classical Wnt-Frizzled signaling leads to the stabilization of cytosolic $\beta$ catenin and activation of Tcf/Lef transcription factors. Increased activity of this pathway owing to mutations in such genes as $t c f-4$ or those for adenomatous polyposis coli or $\beta$-catenin results in the formation of tumors. In many colorectal carcinomas and tumor models Tcf/Lef-responsive genes are upregulated. Based on the hypothesis that the genes targeted by Tcf/Lef might be at risk for producing tumors when activated by a second, alternative signaling pathway, we analyzed their promoter regions for further, putative, tumor-associated cis-acting elements. The kind of analysis performed is based on the assumption that biologically relevant elements are statistically overrepresented compared with random elements. Since such elements might unravel transcription factor-binding sites active in transformed but not in normal cells, they are candidates for artificial cancer-specific promoters, for which there is an urgent need in several gene-therapy projects.

Larson, Garry P.

[34]

\section{Identification of disease gene variants based on gene-gene interactions}

\section{Garry P. Larson, Louis Geller \& Theodore Krontiris}

Beckman Research Institute, City of Hope National Medical Center, Duarte, California, USA

New methods are needed for the identification of pathogenic alleles of candidate genes that may increase cancer susceptibility. Such risk alleles are expected to be of low penetrance and may act alone or modify the effects of other genes. We have developed a method that enriches for pathogenetic disease variants contingent on gene-gene interactions. Candidate gene pairs are chosen based on previous evidence demonstrating genetic or biochemical interaction. Utilizing a cohort of sibling pairs affected with breast cancer, we tested this paradigm by identifying risk variants of cell cycle control gene CDKN1A by means of interactions with TP53 and BRCA1. This approach, which we call disease association by locus stratification, first stratified affected pairs based on sharing of both alleles at a microsatellite marker linked to CDKN1A. The second stratification was based on microsatellite marker sharing at TP53 or BRCA1. We identified subsets of affected pairs sharing both alleles at both loci as screening targets. Utilizing this approach, we were able to enrich for two noncoding disease haplotypes of CDKN1A by virtue of $B R C A 1$ interactions. We defined each haplotype by single-nucleotide polymorphisms at two positions potentially important in CDKN1A transcriptional activation by both p53 and BRCA1p. Our results indicated that an approach based on allele sharing and gene-gene interactions will be valuable not only in identifying risk alleles but also in elucidating their mechanism of action. 